\title{
A Influência de Aspectos Sociais das VANETs na Qualidade de Disseminação de Dados de Eventos Críticos Urbanos
}

\author{
Alisson Yury ${ }^{1}$, Everaldo Andrade $^{2}$, Aldri Santos ${ }^{2}$, Fernando Matos $^{1}$ \\ ${ }^{1}$ Centro de Informática - Universidade Federal da Paraíba (UFPB) \\ ${ }^{2}$ Depto. de Ciência da Computação - Universidade Federal de Minas Gerais (UFMG) \\ alisson.yury@cc.ci.ufpb.br, fernando@ci.ufpb.br, \\ \{everaldo.andrade, aldri\}@dcc.ufmg.br
}

\begin{abstract}
Daily urban critical events demand correct actions by the responsible authorities. Thus, information from these events should be efficiently disseminated to the competent entities. VANETs can naturally support the monitoring of random events in time and space. In addition, social relationships between vehicle users establish local communities whose social intelligence can be applied to disseminate data collected by VANET to support public management in event recognition. This work investigates how social parameters influence the quality of data dissemination of urban critical events. We analyzed the impact of social influence, social activity and friendship strategies, and also consider the tradeoff on QoS and QoE. Results show that social activity brings a more significant influence on $Q o S$, with an increase of $106 \%$ to $175 \%$ in the packet delivery than the others in dense environment. Further, social influence performs better on QoE subjective metrics, delivering packets with an excellent quality level.
\end{abstract}

Resumo. O apoio e o tratamento diário de eventos críticos nas cidades estão entre os grandes desafios dos centros urbanos. Logo, as informações destes eventos devem ser disseminadas eficientemente às entidades competentes. As VANETs naturalmente podem apoiar o monitoramento de eventos aleatórios no tempo e espaço. Além disso, os relacionamentos sociais entre usuários dos veículos estabelecem comunidades locais, cuja inteligência social pode ser aplicada na disseminação dos dados coletados pela VANET a fim de apoiar a gestão pública no reconhecimento de eventos. Este trabalho investiga como os parâmetros sociais influenciam na qualidade de disseminação de dados de eventos críticos urbanos. Analisou-se o impacto das estratégias influência social, atividade social e amizade, e considerou-se os impactos na qualidade de serviço e de experiência. Os resultados mostram que a atividade social tem maior influência na QoS, com aumento de $106 \%$ a $175 \%$ na entrega de pacotes do que as demais em ambientes densos, e a influência social proporciona uma melhor QoE.

\section{Introdução}

O apoio e o tratamento diário de eventos críticos nas cidades estão entre os grandes desafios dos centros urbanos. Esses eventos ocorrem de forma aleatória no tempo e no espaço, e se não tratados eficientemente, podem colocar em risco a integridade das pessoas (incêndios, acidentes, etc) ou simplesmente impactar no bom andamento 
da cidade (e.g. buracos nas vias, engarrafamentos, etc) [Monreal 2018]. As infraestruturas urbanas suportadas pelas Tecnologias da Informação e Comunicação - TICs (redes de comunicação, sensores, atuadores, etc) atuam cada vez mais como facilitador na detecção e disseminação dos eventos urbanos, e as VANETs são o maior expoente devido à ubiquidade dos veículos. A movimentação dos veículos trafegando nas vias urbanas com câmeras e sensores embarcados pode auxiliar na detecção de eventos e as conectividades entre veículos e infraestruturas de redes (V2V e V2I) colaboram para disseminar informações às entidades externas responsável por tratar estes eventos [Andrade et al. 2020]. Além disso, previsões de mercado sugerem que em 2035 existirão 85 milhões de carros conectados por tecnologia 5G [ABI 2020]. A baixa latência e a alta confiabilidade do $5 \mathrm{G}$ integradas à infraestrutura da rede veicular permitirão serviços de comunicação avançados e com maior qualidade. Logo, o tratamento de eventos se beneficiará de serviços de disseminação e entrega mais robustos e confiáveis [Hu et al. 2020].

Contudo, um dos desafios ao provimento da disseminação pelas VANETs ainda consiste na intermitência das conexões pela sua alta dinamicidade topológica [Silva et al. 2016], tal que as soluções de disseminação dependam quase exclusivamente de conexões oportunísticas [Vegni and Loscrí 2015]. Veículos também podem não ter interesse em cooperar na disseminação, criando comportamento egoísta na rede [Nogueira et al. 2014]. Essas questões são desafios ao tratamento de eventos porque sua criticidade exige uma disseminação robusta [Khan and Franzle 2015], tal que a informação chegue rápida e correta à entidade externa. O uso de de técnicas sociais em VANETs têm por um dos princípios que o comportamento dos motoristas no trânsito (como interage com outros motoristas, velocidade, caminhos percorridos, etc) influencia no estabelecimento de relacionamentos entre os veículos [Vegni and Loscrí 2015]. Tais relacionamentos favorecem a cooperação entre os veículos, facilitando alcançarem objetivos em comum, como: - rotinas similares dos motoristas ou proximidade dos veículos podem estabelecer relacionamentos para facilitar o compartilhamento de informações do tráfego em uma região específica; - boa reputação alcançada por veículos permitem que sejam considerados "nós confiáveis" para encaminhar dados sensíveis. Assim, as técnicas sociais contribuem a uma gerência mais inteligente da rede ao possibilitar, por exemplo, escolhas direcionadas de nós mais interessados ou aptos a colaborar [Ullah et al. 2020].

Apesar de existirem trabalhos que consideram aspectos sociais para auxiliar na disseminação de dados em redes veiculares, poucos consideram como as diferentes estratégias sociais impactam na qualidade de experiência, o que é fundamental em situações que necessitam de pronta resposta, como o tratamento de eventos críticos. Geralmente os trabalhos empregam técnicas sociais ao escolher veículos para auxiliar no encaminhamento das mensagens utilizando a influência do veículo na rede [Cunha et al. 2014] ou seus relacionamentos [Campolo et al. 2018]. Outros focam em amenizar o comportamento egoísta dos veículos durante a disseminação utilizando estratégias de reputação [Rahim et al. 2018] ou incentivo [Wang et al. 2018]. Há ainda trabalhos que realizam avaliações de desempenho, porém sem considerar a influência dos aspectos sociais na qualidade [Ullah et al. 2018] ou que se apoiam em uma entidade central [Zia et al. 2020].

Este trabalho investiga como os aspectos sociais influenciam na qualidade de disseminação de dados de eventos críticos urbanos, colaborando para uma melhor qualidade de experiência e de serviço. Ele apresenta uma análise sobre o impacto do com- 
portamento das estratégias influência social, atividade social e amizade na disseminação e entrega dos dados do evento monitorado às autoridades competentes. Assume-se que as autoridades possuem infraestrutura apropriada para processar streams de vídeos, e ao receberem mais informações e mais rápido, são capazes de reconhecer e tratar os eventos de forma mais eficiente. Nesta análise, utilizou-se o sistema SOCIABLE (SOCial monItoring and disseminAtion of urBan criticaL Events) como plataforma para monitoramento e disseminação colaborativa de dados de eventos em VANETs [Yury et al. 2020]. O SOCIABLE opera em uma rede híbrida (V2V e V2I) e leva em conta diferentes relacionamentos estabelecidos ao longo do tempo entre veículos para a criação de comunidades locais. Através da escolha de retransmissores na comunidade, o SOCIABLE faz uma disseminação direcionada (multicast) para encaminhar dados de forma mais ágil, visando o aumento da satisfação do usuário (Quality of Experience - QoE) e a melhora no desempenho da entrega (Quality of Service - QoS). Assim, diferente de outros da literatura, considera-se os impactos na qualidade de serviço e na qualidade de experiência. Resultados mostram que atividade social impacta positivamente na disseminação ao entregar mais pacotes enquanto a influência social proporciona uma melhor percepção do usuário.

O restante do artigo está estruturado como descrito a seguir. A Seção 2 apresenta os trabalhos relacionados. A Seção 3 apresenta os fundamentos de aspectos sociais em VANETs. A Seção 4 descreve o funcionamento do sistema SOCIABLE. A Seção 5 detalha a metodologia de avaliação e os resultados alcançados. Finalmente, a Seção 6 apresenta as considerações finais do trabalho.

\section{Trabalhos Relacionados}

Vários trabalhos na literatura têm utilizado VANETs para tratar ou reduzir as consequências de eventos críticos nos ambientes urbanos. Contudo, a maioria aplica estratégias mais rígidas que dificultam uma seleção inteligente de veículos aptos a colaborar. Já outras soluções baseadas em técnicas sociais empregam abordagens mais dinâmicas levando em conta os laços sociais dos veículos para escolher aqueles mais dispostos a colaborar. Entretanto, muitos não consideram como os aspectos sociais influenciam na qualidade do serviço. Em [Andrade et al. 2020], os autores propõem o sistema MINUET para o monitoramento e disseminação de eventos urbanos críticos baseado em uma abordagem cooperativa de disseminação por agrupamentos. Embora eficaz, devido à estratégia de inundação, ele gera alta redundância de pacotes transmitidos na rede e atraso na entrega, o que pode gerar ineficiência da informação entregue à autoridade dada a criticidade do evento. Além disso, em uma análise em diferentes contextos de tráfego (alta e baixa densidade) e do evento crítico (fixo e móvel) [Andrade et al. 2021], as abordagens de agrupamentos menos dinâmicas impactaram negativamente no monitoramento e disseminação.

Alguns estudos têm investigado o uso de métricas sociais para auxiliar na decisão de disseminação de dados em redes VANETs. Eles focam principalmente no desempenho e dão pouca atenção à experiência do usuário, que é essencial em sistemas de tempo real ou que exige uma sensibilidade para uma pronta resposta como os eventos críticos. Em [Cunha et al. 2014], as trocas de mensagens entre os veículos possibilitam descobrir o grau de cada veículo e seu coeficiente de agrupamento, auxiliando no encaminhamento de mensagens. Embora a solução garanta a entrega das mensagens, em ambientes de baixa densidade, ela compromete a QoS porque gera um alto custo de transmissão devido ao armazenamento e posterior retransmissão de uma grande quantidade de mensagens. 
Já em [Campolo et al. 2018], um framework baseado em Software Defined Networking (SDN) auxilia no estabelecimento de relações sociais entre veículos e facilita a entrega de dados em comunicações Vehicle-to-Everything (V2X). Apesar de na disseminação de alertas o framework obter melhores resultados que o uso da rede celular, ele depende de dispositivos na borda (switches) e de um servidor centralizado.

Outros trabalhos focam no comportamento egoísta dos dispositivos veiculares em redes. Em [Rahim et al. 2018], são aplicadas estratégias sociais (grau de centralidade, conhecimento da comunidade local e global, atividade social e reputação) para conseguir a cooperação entre os veículos e assegurar a retransmissão dos dados. Contudo, eles não mostram como cada técnica influencia nos resultados, sendo que em redes esparsas, os veículos podem ter baixa ou nenhuma atividade social. Além disso, a QoS é prejudicada quando não há um veículo com uma melhor reputação que o veículo retransmissor atual, pois este armazena os dados até que consiga encontrar um veículo com melhor reputação, o que aumenta o custo de transmissão das mensagens. Em [Wang et al. 2018], é proposto um sistema de gerenciamento do tráfego de uma cidade inteligente em situações de eventos anormais utilizando crowdsensing, onde uma técnica de incentivo monetário virtual baseada em créditos encoraja os veículos a cooperarem no encaminhamento de mensagens. Eles utilizaram a estrutura da rede de telefonia junto com as RSUs para um melhor custo-benefício. Entretanto, os veículos podem ajudar na disseminação e não serem recompensados caso a mensagem expire antes de alcançar a RSU, o que faz o sistema não ter conhecimento que o veículo participou na disseminação. Além disso, proporciona uma má experiência ao usuário (motorista) devido ao desperdício de recursos.

Há trabalhos que realizam avaliações de desempenho de estratégias sociais em VANETs comparando-as com outros trabalhos da literatura, porém não mostram como as estratégias sociais influenciam diretamente no desempenho. Em [Ullah et al. 2018] é proposto um esquema de disseminação de mensagens emergenciais em VSN para garantir a segurança dos usuários em situações de emergência usando propriedades sociais dos veículos como, centralidade, amizades e grupos de interesse. No entanto, não há um detalhamento da influência de cada propriedade social e seus respectivos impactos. Já em [Zia et al. 2020], é proposto um modelo baseado em agentes de compartilhamento de informações para recomendações em contexto, a fim de quantificar a satisfação do usuário em respeito à conquista de um objetivo. Ele considera que um mecanismo de aplicação de qualidade melhora o QoE e os resultados validam essa hipótese. Contudo, esta solução depende de uma autoridade central como mecanismo de aplicação de qualidade.

\section{Aspectos Sociais em VANETs}

Esta seção revisa brevemente aspectos sociais que têm sido considerados em VANETs e são utilizados nesta análise. Geralmente as técnicas sociais utilizadas em VANETs partem da premissa que a interação entre os motoristas influenciam no comportamento da rede e podem favorecer a cooperação entre os veículos. Neste contexto, estratégias sociais vêm sendo aplicadas nos mais diversos serviços de rede veiculares, como encaminhamento de pacotes, roteamento, disseminação de dados, sensoriamento, segurança e gerenciamento de tráfego para melhorar a qualidade do serviço entregue. Especificamente no serviço de disseminação de dados, que é o foco desta análise, é comum o uso de técnicas sociais de comportamento, proximidade e similaridade para estimular/incentivar a cooperação entre os veículos e auxiliar na identificação de veículos melhor 
localizados na rede para selecioná-los como retransmissores. Uma seleção mais criteriosa de retransmissores, permite por exemplo, aumentar a taxa de entrega de pacotes e diminuir o tempo de atraso de entrega. A seguir serão descritas brevemente as estratégias de centralidade, atividade social e amizade empregadas em VANETs e utilizadas neste estudo de análise.

A estratégia de centralidade permite escolher veículos com maior influência sobre a rede com base na sua localização ou posição relativa entre os veículos na rede veicular. Dentre as métricas de centralidade mais comuns estão: Grau de centralidade (DC) quantidade de vizinhos a um salto que o veículo possui; Centralidade por aproximação (CC) - distância de um veículo para todos os outros veículos da rede; Centralidade de entrelaçamento (BC) - corresponde a quantas vezes um veículo está no menor caminho entre todos os pares de veículos; Centralidade eigenvector (EC) - indica quantos veículos importantes estão a um salto do veículo atual. A métrica Influência Social (STR) [Nagaraj et al. 2019], que é composta conforme Equação 1, considera as métricas de centralidade de entrelaçamento (BC), por aproximação (CC), grau (DC) e eigenvector (EC) onde cada métrica possui um peso associado (W), sendo que a soma dos quatro pesos é 1 .

$$
S T R_{i}=W_{B C} *(B C)_{i}+W_{C C} *(C C)_{i}+W_{D C} *(D C)_{i}+W_{E C} *(E C)_{i}
$$

A estratégia de atividade social mede a capacidade de um veículo criar relacionamentos com outros veículos que ainda não estiveram em contato na rede. Da mesma forma que acontece na vida social, um indivíduo que encontra novas pessoas diariamente é considerado um indivíduo mais socialmente ativo quando comparado a outros que mantém sempre o mesmo círculo de amizades [Rahim et al. 2018]. Um valor alto de atividade social indica que o veículo é mais ativo na rede e a probabilidade de encontrar novos vizinhos é alta. Consequentemente, a probabilidade de entregar pacotes é maior. A atividade social $S A c t_{i}(t)$ de um veículo $v_{i}$ no instante $t$ é medida conforme a Equação 2, onde $N_{v_{i}}(t)$ e $N_{v_{i}}(t-\Delta t)$ representam o número de vizinhos atuais de $v_{i}$ em $t$ e o número de vizinhos anteriores de $v_{i}$ no instante $t-\Delta t$, respectivamente.

$$
\operatorname{SAct}_{i}(t)=1-\frac{N_{v_{i}}(t-\Delta t) \cap N_{v_{i}}(t)}{N_{v_{i}}(t-\Delta t) \cup N_{v_{i}}(t)}
$$

A estratégia amizade mede a frequência com que os veículos entram em contato entre si. Quando dois veículos se encontram pela primeira vez, é estabelecido um relacionamento fraco entre eles. Caso se encontrem novamente, eles podem ser considerados estranhos familiares [Paulos and Goodman 2004]. A medida que se encontram, o relacionamento vai ficando mais forte até se tornarem amigos. Uma vez amigos, os veículos estarão mais propensos a cooperarem entre si. A amizade é representada conforme a Equação 3 onde $f_{v_{i}, v_{j}}$ corresponde à frequência em que os veículos $v_{i}$ e $v_{j}$ se encontram sendo que a frequência representa a força do relacionamento entre $v_{i}$ e $v_{j}$.

$$
\text { Amizade }_{i, j}=f_{v_{i}, v_{j}}
$$

A Figura 1 mostra exemplos simples das três estratégias sociais mencionadas. Na Figura11(a) - Influência Social, o veículo verde tem o maior número de vizinhos, consegue alcançar todos os outros veículos com menor número de saltos e aparece mais vezes no 

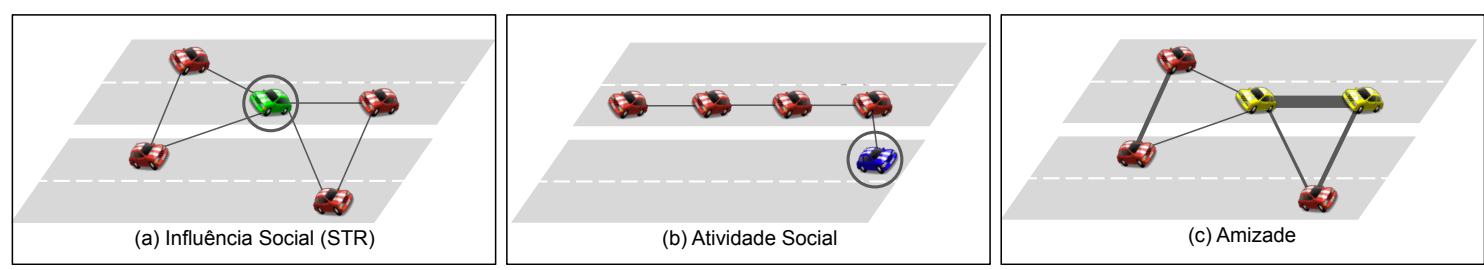

Figura 1. Exemplos de comportamento das estratégias sociais

menor caminho entre todos os outros pares de veículos, possuindo o maior $S T R$ (Eq. 1) e sendo portanto o veículo melhor localizado. Na Figura 1(b) - Atividade Social, o veículo azul entra em contato com todos os outros veículos em vermelho por estar em sentido contrário ao deles, e para os veículos vermelhos, o veículo azul foi o único veículo novo que encontraram, fazendo assim o veículo azul possuir a maior $S A c t(t)$ (Eq. 2). Já a Figura1(c) - Amizade, mostra a força dos relacionamentos sociais entre os veículos. Quanto mais forte, mais provável serem amigos. Neste exemplo, os dois veículos amarelos são amigos pois possuem um relacionamento forte com alto valor de Amizade (Eq. 3).

\section{Visão Geral do Sociable}

O sistema SOCIABLE (SOCial monItoring and disseminAtion of urBan criticaL Event) [Yury et al. 2020] atua no monitoramento dinâmico e disseminação cooperativa de informações de eventos críticos urbanos sob ambientes da Internet Social de Veículos (Social Internet of Vehicles - SIoV) a fim de auxiliar autoridades no tratamento destes eventos. Ele adota uma gerência de organização e controle adaptativos a partir das propriedades sociais e contextos temporal e espacial dos veículos na rede, sendo composto de dois níveis: o Nível Físico (NF), consistindo dos veículos trafegando e comunicando-se entre si (V2V) e com uma infraestrutura (V2I) na malha viária; e o Nivel Social (NS), consistindo na coordenação dos perfis sociais dos veículos, construídos com base em propriedades sociais (e.g. rotina similar, interesse), o que permite a formação de comunidades por meio da similaridade das propriedades sociais a fim de prover serviços cooperativos para auxiliar autoridades no tratamento do evento.

O SOCIABLE suporta diferentes estratégias sociais empregadas para seleção de veículos retransmissores. Estas estratégias levam em conta diferentes aspectos sociais, providos pelos veículos da comunidade, o que incentiva a cooperação dinâmica entre veículos adequada aos contextos de tráfego e eventos de naturezas distintas, como incêndios, acidentes, engarrafamentos, entre outros. A Figura 2 ilustra as estratégias sociais empregadas no SOCIABLE: 1) influência social, 2) atividade social e 3) amizade, sob diferentes contextos de tráfego. Cada estratégia adota uma abordagem social diferente que o SOCIABLE utiliza para selecionar veículos retransmissores oportunos a fim de coordenar a disseminação das informações do evento crítico. Cada uma das estratégias influencia a qualidade de transmissão dos streams de vídeo em termos de QoS e QoE do usuário, o que pode induzir o reconhecimento do evento pelas autoridades.

A Figura 3 descreve a interação entre veículos e estações-base (EBs) durante o funcionamento do SOCIABLE, permitindo que cada veículo $V_{i}$ assuma múltiplos papéis dependendo da posição relativa ao evento, vizinhos e EBs. Assim, um veículo que detecta um evento crítico $(E v)$ atua como detector $\left(V_{d}\right)$. Se estiver ao alcance de uma EB e for capaz de repassar mensagens entre vizinhos, também pode atuar como Gateway $(G w)$ e 


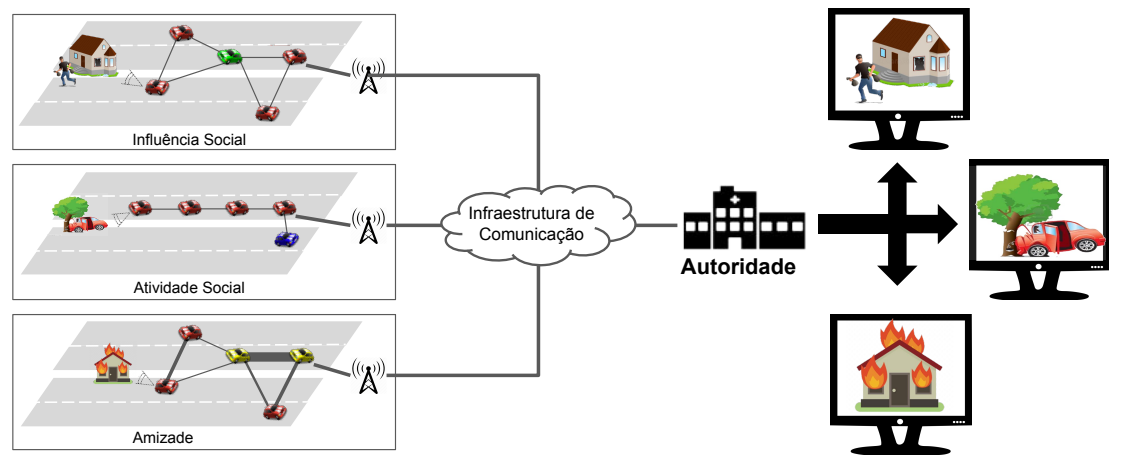

Figura 2. Estratégias sociais empregadas no SOCIABLE

retransmissor $\left(V_{r}\right)$, respectivamente. Inicialmente, cada veículo $V_{i}$ troca continuamente mensagens de Beacon (msg.Beacon) com seus vizinhos para reconhecer a comunidade a qual pertence através de uma estratégia de reconhecimento de comunidade baseado na rotina similar dos veículos [Cunha et al. 2016]. Ao detectar $E v$, o veículo $V_{d}$ coleta informações de contexto de $E v(m s g$. Cont $(E v))$ e gera mensagens de monitoramento (msg.Mon $(E v)$ ) com um tempo de vida (Time to Live - TTL) para cada mensagem. Através do controle de disseminação do SOCIABLE, $V_{d}$ inicia a transmissão de $m s g$.Mon( $\left.E v\right)$ para a rede. Estando no alcance de uma EB, ele oportunamente se auto define $G w$ e entrega as $m s g . M o n(E v)$ à EB, que por sua vez, encaminha a mensagem à autoridade (Aut). Em seguida, ele dissemina as $m s g . M o n(E v)$ para cada veículo $V_{i}$ que pertence à sua comunidade local. Contudo, antes de transmitir msg.Mon $(E v), V_{d}$ aplica uma das estratégias sociais empregadas no SOCIABLE (influência social, atividade social ou amizade), a fim de selecionar o próximo veículo retransmissor $\left(V_{r}\right)$, e agrega esta informação à $m s g$.Mon $(E v)$.

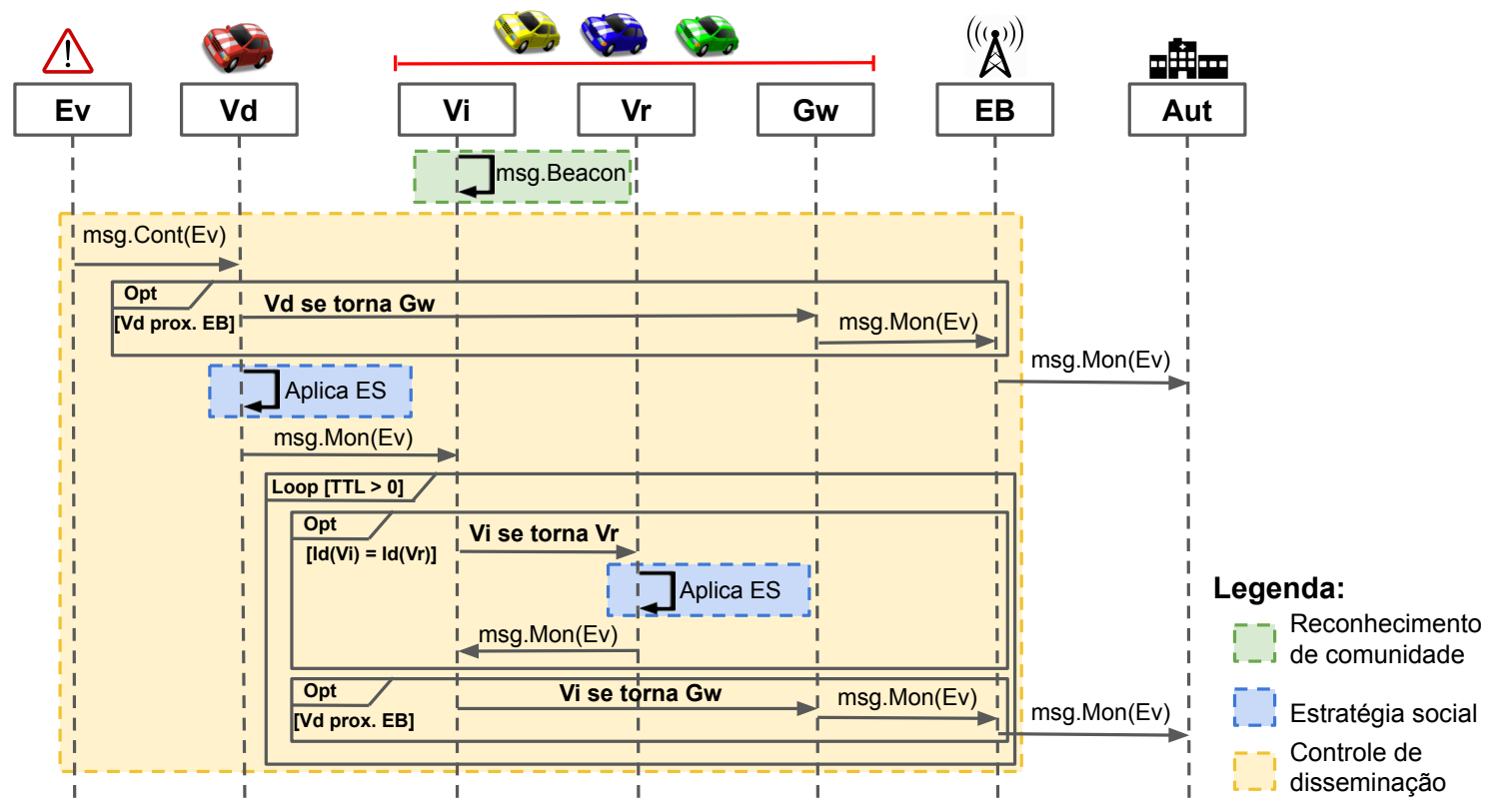

Figura 3. Troca de mensagens entre entidades do SOCIABLE

Quando $V_{i}$ recebe uma msg.Mon(Ev), ele verifica se o TTL expirou (TTL=0). Caso não tenha expirado e se $V_{i}$ for o próximo $V_{r}\left(\operatorname{Id}\left(V_{i}\right)=\operatorname{Id}\left(V_{r}\right)\right)$, então ele assume o papel de $V_{r}$, aplica a estratégia social, seleciona próximo $V_{r}$ e dissemina $m s g . M o n(E v)$ para comunidade. Se $V_{i}$ estiver ao alcance de uma EB, ele se torna $G w$ e entrega msg.Mon(Ev) à EB, que será responsável por encaminhá-la à autoridade competente. Caso TTL tenha 
expirado, as mensagens recebidas serão descartadas o que aumenta a precisão de contexto do evento entregue às autoridades e reduz a carga de mensagens transmitidas na rede.

A Figura 4 exemplifica o funcionamento do SOCIABLE em um ambiente urbano onde uma comunidade de veículos colabora no monitoramento e disseminação de informações multimídia de um evento crítico até uma estação base. O cenário corresponde a duas vias de faixa dupla e sentidos opostos composto por um conjunto de veículos $V=\{v 1, v 2, v 3, v 4, v 5, v 6, v 7, v 8\}$, uma estação base $E B$, um evento crítico $E v$ ocorrendo em uma das vias e uma comunidade $C_{1} \subseteq V$ composta pelos veículos azuis. No instante de tempo $t 1$, ao detectar o evento $E v$, o veículo $v 1$ simultaneamente inicia a seleção do veículo retransmissor, pela aplicação da estratégia social, e o monitoramento, criando assim o pacote de monitoramento $P$ com um $T T L=3$, definindo o número máximo de transmissões de $P$. Assumindo que o SOCIABLE utilize a amizade como estratégia social, $v 1$ conclui que, dentre seus vizinhos, $v 2$ é o melhor candidato a retransmissor em razão do seu relacionamento ser mais forte com $v 1$ dentre os demais. Assim, o pacote $P$ é transmitido por $v 1$, com as informações de $E v$ e o próximo retransmissor (i.e. $v 2$ ), para a comunidade $C 1$. Considerando que a cada transmissão de $P$, o seu $T T L$ diminui em uma unidade, $v 2$ e $v 6$ ainda podem retransmistir $P$ pois seu $T T L=2$.

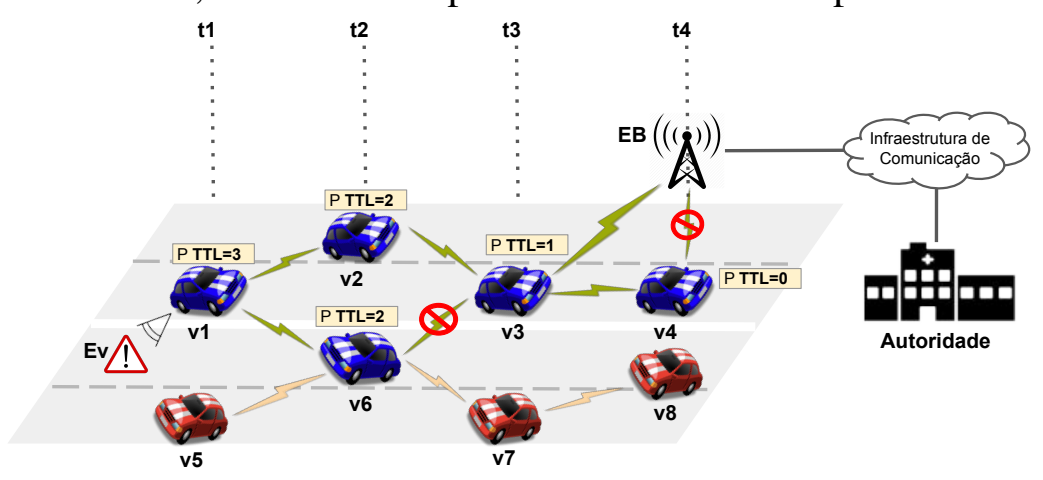

Figura 4. Exemplo de operação do SOCIABLE

No instante $t 2$, os veículos $v 2$ e $v 6$ recebem $P$. Neste caso, $v 2$ reconhece que é retransmissor, então seleciona próximo retransmissor, através do calculo de amizade com seus vizinhos, define $v 3$ como retransmissor e repassa $P$ com o novo retransmissor para $C 1$. Como $v 6$ não foi selecionado como retransmissor e não está próximo de $E B$, ele não repassa $P$. No instante $t 3$, o veículo $v 3$ recebe $P$. Como ele está próximo de $E B$ (Gateway) e o $T T L$ de $P$ não expirou, $v 3$ repassa $P$ para $E B$, a qual repassará $P$ à autoridade. Ademais, como $v 3$ também é retransmissor, ele conclui que $v 4$ possui uma maior relação de amizade e o define como retransmissor. Em seguida, $v 3$ repassa $P$ para sua comunidade. Ao receber $P$, em $t 4$, o veículo $v 4$ verifica que é retransmissor e está próximo de $E B$. Contudo, ele descarta o pacote em razão do $T T L$ expirado (i.e. $T T L=0$ ).

\section{Metodologia e Análise}

Esta seção descreve a metodologia de implementação e de avaliação e análise do SOCIABLE $]^{1}$ através de experimentos simulados sob modelos de ambientes urbanos realísticos. Verificou-se a qualidade de serviço provido pelo SOCIABLE, sob cada estratégia social empregada (Influência Social (STR), Atividade Social (AS) e Amizade

\footnotetext{
${ }^{1}$ Disponível para solicitação em: https://bitbucket.org/everaldoAndrade/sociable
} 
(AM)), em termos de eficácia e eficiência dos serviços de monitoramento, disseminação e entrega das informações de eventos fixos às EBs. O SOCIABLE foi implementado em C++ e executado no simulador NS3, versão 3.28, com o apoio do SUMO. O NS3 permite simulações mais próximas da realidade, já que contempla diversas características dos ambientes de redes, como interferência no meio. As simulações foram realizadas em dois cenários (alta densidade (AD) e baixa densidade (BD)), cada um usando uma estratégia social diferente em uma máquina de 23.5GB de RAM com CPU Intel(R) Xeon(R) Silver 4114 rodando Ubuntu 18.04.5 LTS. O ambiente de avaliação consiste de uma região do LuST (Luxembourg SUMO Traffic) $)^{2}$ e representa a malha viária com padrões de tráfego urbano da cidade de Luxemburgo. A Figura 5 mostra o trecho da malha viária urbana selecionado para execução dos testes. A avaliação consistiu na operação do SOCIABLE sob o ambiente simulado, no qual foram realizadas simulações para cada uma das estratégias sociais em cenários de baixa densidade (entre 05hs:30min e 05hs:35min) e alta densidade (entre 08hs:00min e 08hs:05min), buscando assim contextos de tráfegos com baixa conectividade e com alta sobrecarga de pacotes transmitidos na rede, respectivamente. $\mathrm{O}$ cenário possui duas estações-base e um evento fixo com duração de quatro minutos. A Tabela 1 apresenta os parâmetros de configuração definidos para a avaliação.

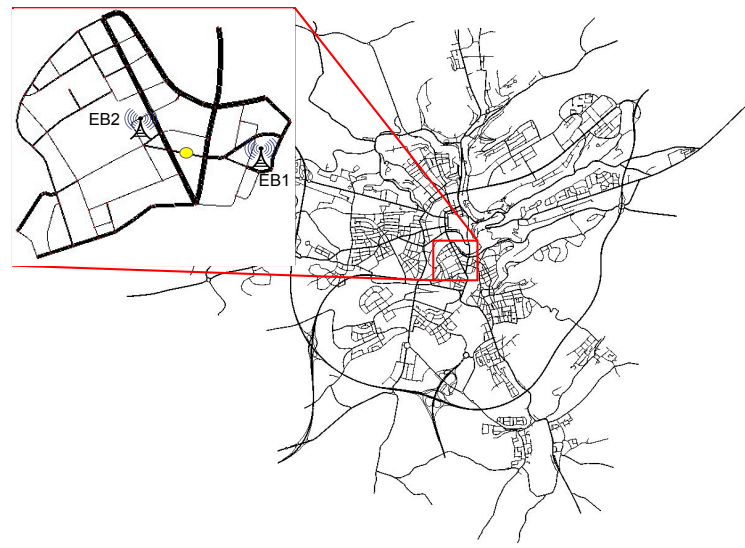

Figura 5. Cenários
Tabela 1. Parâmetros das simulações

\begin{tabular}{lll}
\hline Parâmetro & BD & AD \\
\hline Tempo da simulação & 5 mins & 5 mins \\
Número de eventos & 1 & 1 \\
Duração do evento & 4 mins & 4 mins \\
Característica das vias & Zona urbana & Zona urbana \\
Raio de transm. & $100 \mathrm{~m}$ & $100 \mathrm{~m}$ \\
Número de veículos & 219 & 995 \\
Número de EBs & 2 & 2 \\
Dist. das EBs ao evento & EB1: 220m e EB2: 194m \\
\hline \hline Video & \multicolumn{2}{c}{ Highway (2000 frames)* } \\
Video frame size & $352 \times 288$ pixels (CIF format) \\
Frame rate & \multicolumn{2}{c}{ 24 frames/second } \\
Enconding & \multicolumn{2}{c}{ H.264/MPEG-4 AVC } \\
Bitrate & \multicolumn{2}{c}{ 768 kbs } \\
YUV format & 4:0 \\
*Disponível em: http://trace.eas.asu.edu/yuv/
\end{tabular}

A avaliação em termos de QoS do SOCIABLE foi realizada aferindo métricas que avaliam a eficácia (Número de pacotes de monitoramento criados (Npmc) e Número de pacotes de monitoramento entregues (Npme)) e a eficiência (Número de veículos que colaboram $(N v c)$, Taxa de pacotes entregues (Tpe) e Atraso médio de entrega (Ame)) do monitoramento e disseminação das informações. Em termos de QoE, foram aferidas as seguintes métricas comumente utilizadas na literatura: Pico da relação sinal ruído médio (Peak Signal to Noise Ratio - PSNR, Índice de similaridade estrutural médio (Structural Similarity Index Method - SSIM) e Pontuação de opinião média (Mean Opinion Score $M O S$ ). Utilizamos EvalVid ${ }^{3}$ para aferir as métricas de QoE. A Tabela2 2 define as métricas de avaliação utilizadas e suas respectivas fórmulas.

\footnotetext{
${ }^{2}$ Versão 2.0 disponível em: https://github.com/lcodeca/LuSTScenario

${ }^{3}$ Versão 2.7 disponível em: https://www.tkn.tu-berlin.de/research/evalvid/
} 
Tabela 2. Descrição e fórmula das métricas aferidas

\begin{tabular}{|c|c|c|}
\hline \multirow{10}{*}{ QoS } & \multirow[t]{2}{*}{$N v c(\Delta t)$} & $\begin{array}{l}\text { Corresponde ao número de veículos da malha viária }(N(\Delta t)) \text { que colaboram (i.e. monitoram e/ou } \\
\text { disseminam) em um intervalo de tempo } \Delta \mathrm{t} \text {. Mostra o nível de colaboração do SOCIABLE durante a } \\
\text { ocorrência de um evento. }\end{array}$ \\
\hline & & $\sum_{i=1}^{N(\Delta t)} 1, \forall$ veículo $v_{i}$ onde colabo $\left(v_{i}\right)=$ verdadeiro \\
\hline & \multirow[t]{2}{*}{ Npmc } & $\begin{array}{l}\text { Denota a capacidade de monitoramento do SOCIABLE, dado pela soma do número de pacotes criados } \\
\left(P M_{c r i a}\right) \text { por cada veículo } v_{i} \text { que detecta o evento }(N v d) \text {. Afere a eficácia de detecção de eventos. }\end{array}$ \\
\hline & & $\sum_{i=1}^{N v d}\left(P M_{\text {cria }}\left(v_{i}\right)\right)$ \\
\hline & \multirow[t]{2}{*}{ Npme } & $\begin{array}{l}\text { Corresponde à capacidade de entrega de pacotes de monitoramento às autoridades competentes, dado } \\
\text { pela soma de pacotes entregues em cada EB dentre os pacotes transmitidos } N_{p} \text { na rede. Afere a eficácia } \\
\text { das estratégias sociais na entrega de pacotes às EBs. }\end{array}$ \\
\hline & & $\sum_{i=1}^{N_{p}} 1, \forall$ pacote $p_{i}$ entregue à uma EB \\
\hline & \multirow[t]{2}{*}{ Tpe } & $\begin{array}{l}\text { Corresponde à taxa de pacotes entregues, dada pela razão de pacotes de monitoramento não duplicados } \\
\text { (i.e pacotes recebidos não repetidos) entregues nas EBs }\left(P M_{\text {entregues }}\right) \text { pelo total de pacotes criados } \\
\left(P M_{\text {criados }}\right) \text {. Afere a eficiência das estratégias sociais na entrega de pacotes às EBs. }\end{array}$ \\
\hline & & $\frac{P M_{\text {entregues }}}{P M_{\text {criados }}} * 100$ \\
\hline & \multirow[t]{2}{*}{ Ame } & $\begin{array}{l}\text { Indica o atraso médio dos pacotes de monitoramento entregues às EBs, dado pela soma total dos inter- } \\
\text { valos de tempo entre a criação }\left(T_{\text {criação }}\right) \text { e entrega }\left(T_{\text {entrega }}\right) \text { de cada pacote } p_{i} \text {, pelo total de pacotes } \\
\text { entregues } P M_{\text {entregues. Quanto menor o atraso, maior a eficiência da estratégia social na entrega. }}\end{array}$ \\
\hline & & $\frac{\sum_{i=1}^{N}\left(T_{\text {criação }}\left(p_{i}\right)-T_{\text {entrega }}\left(p_{i}\right)\right)}{P M_{\text {entregues }}}$ \\
\hline \multirow{6}{*}{ QoE } & \multirow[t]{2}{*}{$P S N R_{a v g}(\Delta t)$} & $\begin{array}{l}\text { Corresponde ao PSNR médio dos frames entregues no intervalo } \Delta t(\mathrm{~F}(\Delta t)) \text {, dado pela soma do valor } \\
\text { PSNR de cada frame } f_{i}\left(\operatorname{PSNR}\left(f_{i}\right)\right) \text { pelo total de frames entregues }\left(F_{\text {entregues }}\right) \text { em } \Delta t .\end{array}$ \\
\hline & & $\frac{\sum_{i=1}^{F(\Delta t)}\left(P S N R\left(f_{i}\right)\right.}{F_{\text {entregues }}}$ \\
\hline & \multirow[t]{2}{*}{$\operatorname{SSIM} M_{a v g}(\Delta t)$} & $\begin{array}{l}\text { Corresponde ao SSIM médio dos frames entregues no intervalo } \Delta t(\mathrm{~F}(\Delta t)) \text {, dado pela soma do valor } \\
\text { SSIM de cada frame } f_{i}\left(\operatorname{SSIM}\left(f_{i}\right)\right) \text { pelo total de frames entregues }\left(F_{\text {entregues }}\right) \text { em } \Delta t \text {. }\end{array}$ \\
\hline & & $\frac{\sum_{i=1}^{F(\Delta t)}\left(S S I M\left(f_{i}\right)\right.}{F_{\text {entregues }}}$ \\
\hline & \multirow[t]{2}{*}{ MOS } & $\begin{array}{l}\text { Mensura a qualidade subjetiva da percepção do usuário e mapeia o nível de satisfação em uma escala } \\
\text { de } 1 \text { (Ruim) a } 5 \text { (Excelente). A porcentagem de cada nível } i \text { é dado pelo razão dos frames de nível } i \\
\left(M O S_{i}\right) \text { pelo total de frames entregues }\left(F_{\text {entregues }}\right) \text {. }\end{array}$ \\
\hline & & $\frac{M^{\prime} O S_{i}}{F_{\text {entregues }}} * 100$ \\
\hline
\end{tabular}

\subsection{Resultados}

Esta seção apresenta as análises dos testes realizados para avaliar o desempenho e a experiência do usuário de cada estratégia social empregada no SOCIABLE. Os gráficos da Figura 6 mostram o número de veículos colaborando ao longo do tempo (Nvc $(\Delta t)$ ) em cada uma das estratégias sociais nos cenários de alta $(\mathrm{AD})$ e de baixa densidade (BD). Em ambos os cenários, há cooperação de veículos em todas as estratégias sociais empregadas pelo SOCIABLE para disseminação de dados do evento detectado. No entanto, há mais veículos colaborando na estratégia de Atividade Social (AS) (Gráficos 6(b), 6(e)) em relação às estratégias Influência Social (STR) e Amizade (AM) (Gráficos 6(a), 6(d) e 6(c), 6(f), respectivamente), pois a AS seleciona veículos que conhecem novos vizinhos e perdem contato com vizinhos antigos com maior frequência.

Os gráficos da Figura 7 mostram o atraso médio de entrega (Ame) em relação ao número de saltos de cada pacote entregue. No cenário BD (Gráfico 7(a)), a STR apresenta Ame menor que a AS devido selecionar o veículo melhor localizado na rede como próximo retransmissor entregando pacotes com 3 saltos. Entretanto, a AS entrega pacotes com apenas 2 saltos devido à maior facilidade de encontrar veículos gateways. No cenário AD (Gráfico 7(b)), a AS apresenta Ame menor que as outras estratégias por existirem mais gateways na rede. Em contrapartida, a AM tem Ame maior no cenário AD e não entrega pacotes no cenário $\mathrm{BD}$, pois selecionar veículos com relacionamentos fortes 

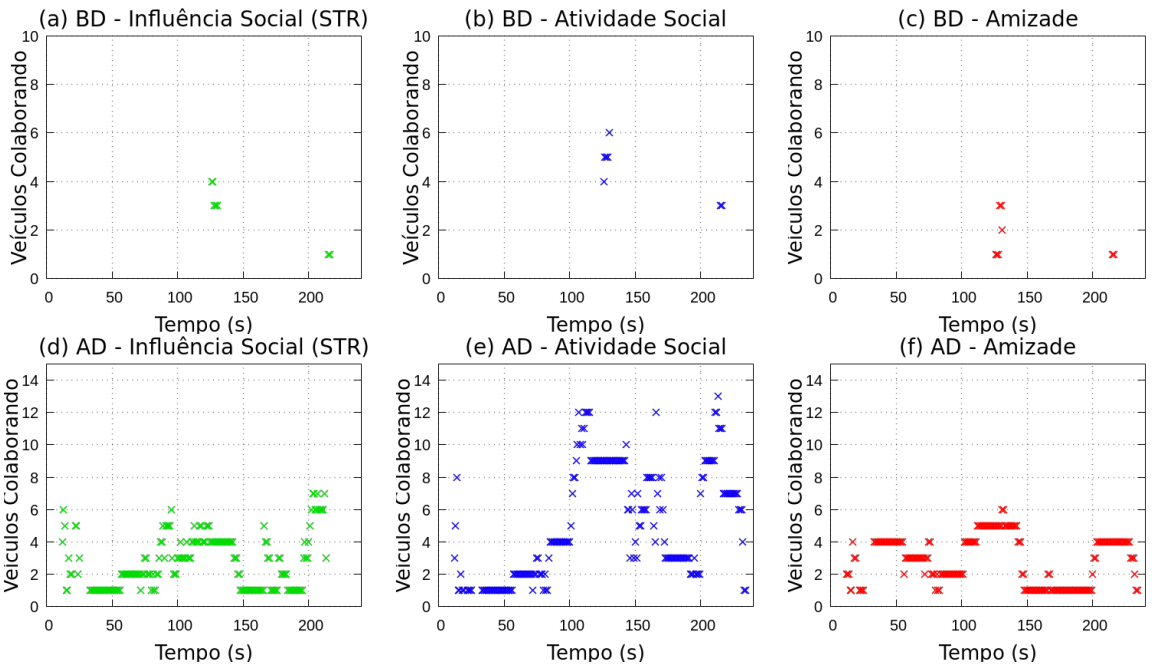

Figura 6. Veículos colaborando no SOCIABLE

como retransmissores não é o mais adequado para disseminar dados de maneira eficiente. Estes resultados mostram as capacidades da AS entregar pacotes rapidamente com poucos saltos e da STR selecionar veículos melhor localizados na rede como retransmissores.
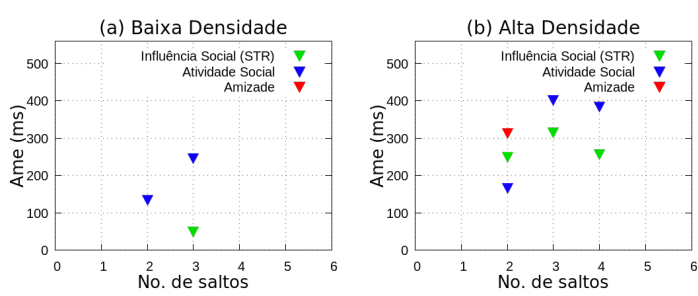

Figura 7. Ame X No. de saltos
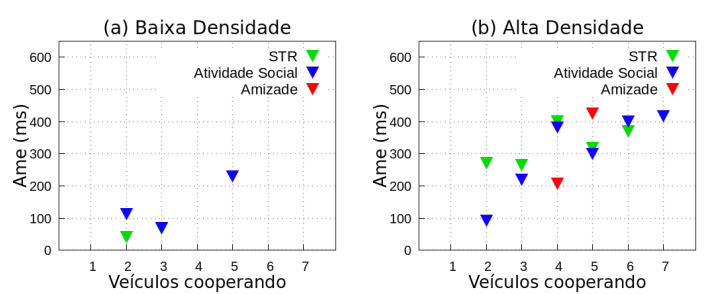

Figura 8. Ame X NVC

Os gráficos da Figura 8 exibem o atraso médio de entrega (Ame) de pacotes de monitoramento gerados pelo número de veículos que colaboram para a entrega em cada estratégia social. Nos cenários BD e AD (Gráficos 8(a) e 8(b)), uma quantidade maior de veículos colaboram na AS para entregar pacotes. No cenário $\mathrm{BD}$, a STR possui Ame menor com menos da metade de veículos colaborando que na AS. No cenário AD, a AM obteve Ame menor que as demais com 4 veículos colaborando, porém possui Ame maior com 5 veículos colaborando. A STR possui Ame semelhante independente do número de veículos colaborando. Estes resultados mostram que a AS apresenta Ame menor que as demais estratégias, porém esta diferença diminui conforme há mais veículos colaborando.

Os gráficos da Figura 9 apresentam a quantidade de pacotes de monitoramento criados (NPMC) quando o evento é detectado e de pacotes entregues (NPME) à EB de acordo com o número de veículos colaborando (NVC) no SOCIABLE. Nos cenários BD e $\mathrm{AD}$, as estratégias sociais possuem NPMC iguais porque há o mesmo número de veículos detectores. No cenário BD, a AM (Gráfico 9(c)) não entrega pacotes, porém consegue entregar no cenário $\mathrm{AD}$ (Gráfico 9 (f)). As demais estratégias entregam pacotes em ambos cenários. Estes resultados indicam que as estratégias AS e STR usadas pelo SOCIABLE detectam, monitoram e entregam pacotes de monitoramento de eventos urbanos às EBs em diferentes condições de ambiente, e a AM também detecta e monitora eventos urbanos em diferentes condições de ambiente, mas só entrega pacotes em ambientes densos. 

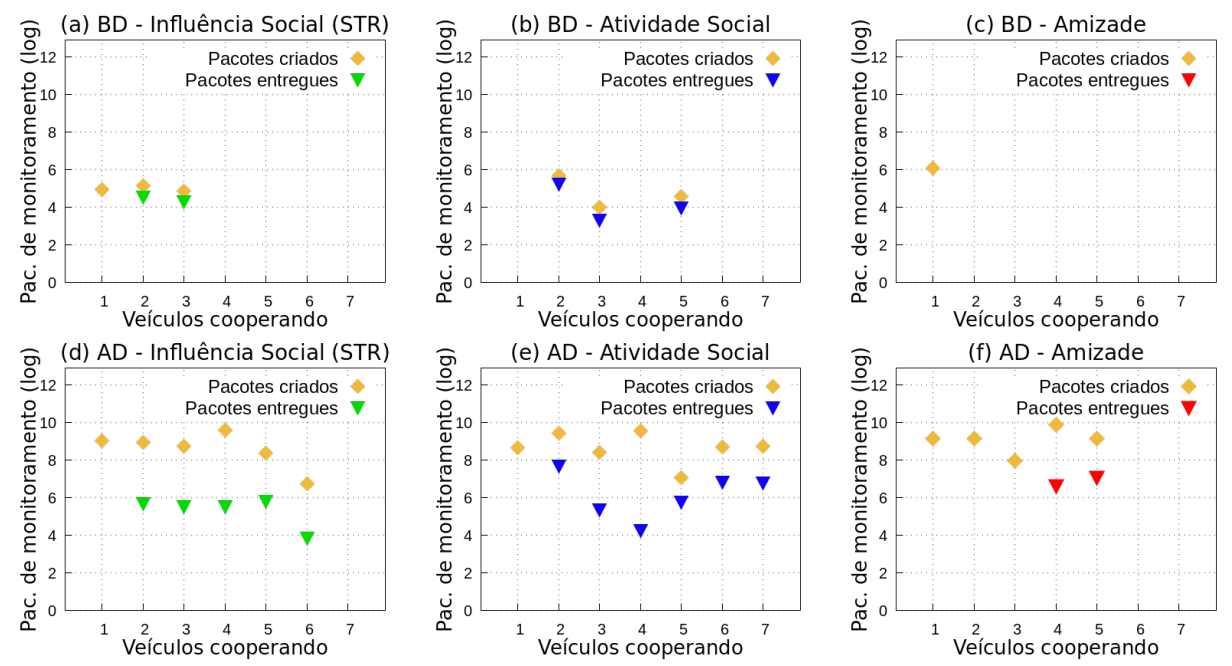

Figura 9. Qtde de pacotes criados e entregues às EBs no SOCIABLE

O gráfico da Figura 10 mostra a taxa de pacotes de monitoramento não-duplicados entregues às EBs pelo total de pacotes criados (Tpe). No cenário BD (Gráfico 10(a)), como foi comentado anteriormente, a AM não entrega pacotes. A AS entrega mais pacotes que a STR alcançando Tpe de $35 \%$ enquanto a STR possui Tpe de $22 \%$. Já no cenário AD (Gráfico 10(b)), a AS entrega $7,65 \%$ dos pacotes, com aumento de $175 \%$ e

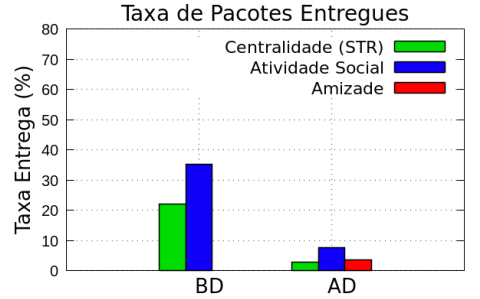

Figura 10. Taxas de pacotes entregues às Estações-Bases $106 \%$ em relação à STR e à AM, respectivamente. Estes resultados mostram que AS consegue entregar pacotes em diferentes condições de ambiente, enquanto a AM entrega apenas em ambientes com alta densidade.

Os gráficos da Figura 11 mostram o PSNR médio dos frames de vídeo do evento urbano entregues pelo SOCIABLE utilizando as estratégias sociais STR, AS e AM nos cenários BD e AD. No cenário BD (Gráficos 11(a) e 11(b)) as estratégias STR e AS, respectivamente, possuem o valor de PSNR médio de $10 \mathrm{~dB}$ que indica uma qualidade de reconstrução de vídeo baixa para a percepção humana. Além da baixa densidade prejudicar a disseminação das informações do evento, esse valor de PSNR baixo pode ser explicado devido à distância entre o local de ocorrência do evento e a posição das EBs. No cenário $\mathrm{AD}$ (Gráficos 11(c-e)), apesar das distâncias entre o evento e às EBs serem as mesmas que no cenário BD, uma maior quantidade de veículos entrega mais pacotes, melhorando o valor de PSNR médio. A STR alcança PSNR médio com qualidade de reconstrução de vídeo favorável para a percepção humana, entre [25-40]dB, no início da simulação. Já a AS alcança PSNR médio com qualidade de reconstrução de vídeo favorável para a percepção humana em dois momentos. A AM mantém o PSNR médio próximo de $10 \mathrm{~dB}$, não tendo um valor de PSNR aceitável durante a simulação.

A Figura 12 mostra gráficos com a similaridade estrutural dos frames de vídeo entregues pelo total de frames entregues (SSIM) em ambos os cenários para as estratégias utilizadas. Valores de SSIM entre $[0,5,1]$ são aceitáveis para comunicações sem fio. Os Gráficos 12(a) e (b) mostram o SSIM das estratégias STR e AS, respectivamente, no 

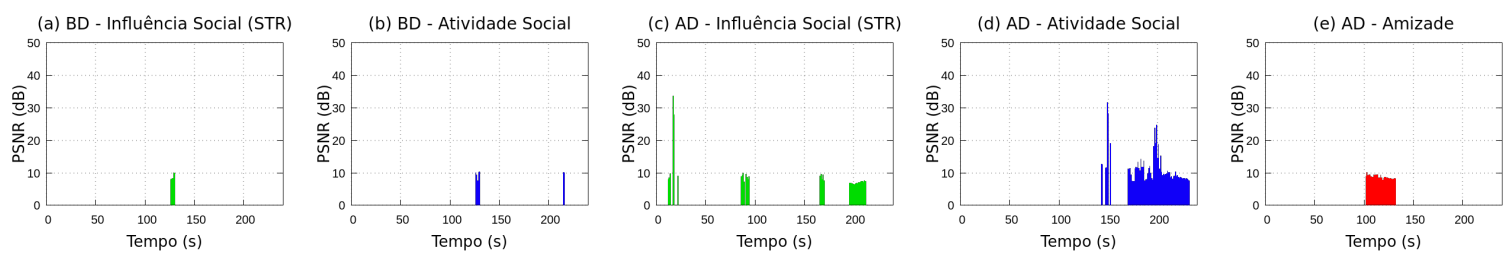

Figura 11. PSNR médio de frames entregues ao longo do tempo

cenário BD. Pode-se notar que ambas estratégias entregam frames de vídeo com valores de SSIM entre $[0,5,1]$, o que indica uma qualidade de vídeo adequada. No cenário AD, a AM (Gráfico 10(e)) entrega frames com SSIM indicando uma qualidade de vídeo adequada. Já a STR e AS (Gráficos 12(c) e 12(d)) permanecem no intervalo relevante a maior parte do tempo, com picos de SSIM de 0,9, sendo que em alguns intervalos de tempo não entregam frames com valores de SSIM dentro aceitável $[0,5,1]$.
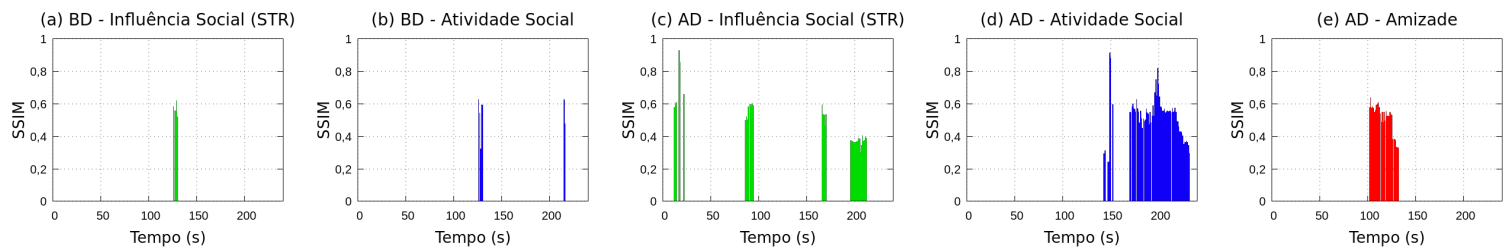

Figura 12. SSIM médio de frames entregues ao longo do tempo

No contexto de disseminação de streaming de vídeo, a correlação entre valores de SSIM e a percepção subjetiva não é sempre precisa. A Figura 13 mostra a métrica subjetiva MOS para vídeos entregues durante o monitoramento do evento urbano. Devido à distância do evento às EBs, as estratégias tiveram em sua grande maioria, níveis ruins de qualidade de vídeo à percepção humana em ambos os cenários. No cenário $\mathrm{AD}$, a STR e a AS entregaram vídeos com qualidade razoável e pobre de acordo com a percepção humana e apenas a STR entregou vídeos com qualidade boa ou excelente.

(a) BD - Esc. MOS

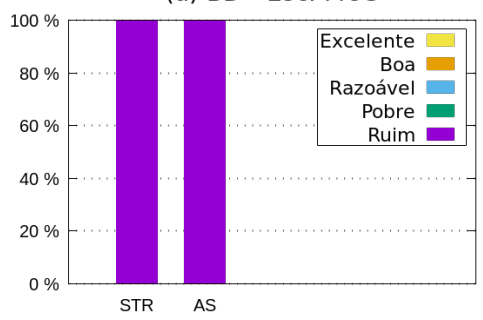

(b) AD - Esc. MOS

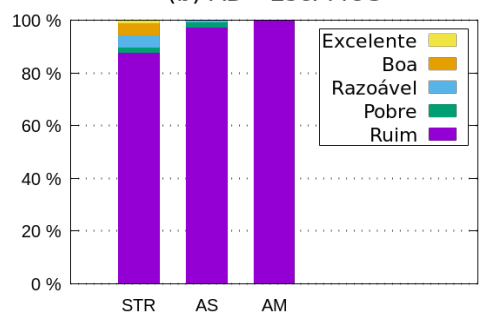

Figura 13. Escala MOS de frames entregues pelas estratégias sociais

\section{Conclusões}

Este trabalho apresentou uma análise dos aspectos sociais na qualidade de disseminação de dados de eventos críticos, apoiado em um ambiente de rede veicular, a fim de auxiliar as autoridades no tratamento do evento. Usamos o sistema SOCIABLE para propagar informações do evento de maneira cooperativa até as autoridades competentes considerando aspectos sociais para seleção de veículos retransmissores. Resultados da simulação mostraram que a utilização de atividade social resulta em uma melhor qualidade do serviço de disseminação, o que é essencial em contextos de eventos críticos. Por outro lado, a influência social obteve melhores resultados em fatores que impactam 
na qualidade de experiência, o que sugere sua utilização em aplicações que dependam da percepção do usuário. Como trabalhos futuros, analisaremos o SOCIABLE diante de eventos sobrepostos e que variam no tempo e espaço ao utilizar novas estratégias sociais.

\section{Referências}

ABI (2020). 5g in automotive and smart transportation. https://www.abiresearch.com/ market-research/product/1033746-5g-in-automotive-and-smart-transportation/.

Andrade, E., Santos, A., Maciel, P. D., and Matos, F. (2021). Analyzing cooperative monitoring and dissemination of critical mobile events by vanets. Wireless Networks, 67:101244.

Andrade, E., Veloso, K., Vasconcelos, N., Santos, A., and Matos, F. (2020). Cooperative monitoring and dissemination of urban events supported by dynamic clustering of vehicles. Pervasive and Mobile Computing, 67:101244.

Campolo, C., Molinaro, A., and Iera, A. (2018). A reference framework for social-enhanced Vehicle-toEverything communications in 5G scenarios. Computer Networks, 143:140-152.

Cunha, F. D., Alvarenga, D. A., Maia, G., Viana, A. C., Mini, R. A., and Loureiro, A. A. (2016). Exploring interactions in vehicular networks. In Proceedings of the 14th ACM MobiWac, pages 131-138.

Cunha, F. D., Maia, G. G., Viana, A. C., Mini, R. A., Villas, L. A., and Loureiro, A. A. (2014). Socially inspired data dissemination for vehicular ad hoc networks. pages 81-85.

Hu, J., Chen, C., Qiu, T., and Pei, Q. (2020). Regional-centralized content dissemination for ev2x services in 5g mmwave-enabled iov. IEEE Internet of Things Journal, 7(8):7234-7249.

Khan, S. and Franzle, M. (2015). Robust mid-range communication in Urban VANETs. In International Conference on Advanced Communication Technology, ICACT, pages 115-120.

Monreal, C. O. (2018). Future urban mobility group: Smart cities research institute swinburne university of technology, Melbourne Australia. IEEE Intelligent Transportation Systems Magazine, 10(2):203-205.

Nagaraj, K., Bhasale, S. S., McNair, J., and Helmy, A. (2019). Vulnerability assessment and classification based on influence metrics in mobile social networks. In the 17th ACM MobiWac, page 9-16. ACM.

Nogueira, M., Arieta, F., Barabasz, L., and Santos, A. (2014). Mitigating flooding attacks on mobility in infrastructure-based vehicular networks. IEEE Latin America Transactions, 12(3):475-483.

Paulos, E. and Goodman, E. (2004). The familiar stranger: Anxiety, comfort, and play in public places. In Proceedings of the SIGCHI Conference on Human Factors in Computing Systems, CHI '04, page 223-230, New York, NY, USA. Association for Computing Machinery.

Rahim, A. et al. (2018). Cooperative data forwarding based on crowdsourcing in vehicular social networks. Pervasive and Mobile Computing, 51:43-55.

Silva, C., Nogueira, M., Kim, D., Cerqueira, E., and Santos, A. (2016). Cognitive radio based connectivity management for resilient end-to-end communications in vanets. Computer Communications, 79:1-8.

Ullah, N. et al. (2018). A Social Utility-Based Dissemination Scheme for Emergency Warning Messages in Vehicular Social Networks. The Computer Journal, 61(7):971-986.

Ullah, N., Kong, X., Ning, Z., Tolba, A., Alrashoud, M., and Xia, F. (2020). Emergency warning messages dissemination in vehicular social networks: A trust based scheme. Vehicular Communications, 22:100199.

Vegni, A. M. and Loscrí, V. (2015). A survey on vehicular social networks. IEEE Communications Surveys and Tutorials, 17(4):2397-2419.

Wang, X. et al. (2018). A City-Wide Real-Time Traffic Management System: Enabling Crowdsensing in Social Internet of Vehicles. IEEE Communications Magazine, 56(9):19-25.

Yury, A., Andrade, E., Nogueira, M., Santos, A., and Matos, F. (2020). Social-based cooperation of vehicles for data dissemination of critical urban events. In GLOBECOM 2020, pages 1-6.

Zia, K., Shafi, M., and Farooq, U. (2020). Improving recommendation accuracy using social network of owners in social internet of vehicles. Future Internet, 12(4). 